\title{
Using Syndromic Surveillance to Investigate Tattoo- related Skin Infections in NYC
}

\author{
Mollie Kotzen*1, ${ }^{\star}$, Robert Mathes ${ }^{1}$, Lillian Lee ${ }^{1}$ and Don Weiss ${ }^{1}$ \\ ${ }^{1}$ Bureau of Communicable Disease, New York City Department of Health and Mental Hygiene, Long Island City, NY, USA; ${ }^{2}$ New York \\ University, New York City, NY, USA
}

\section{Objective}

To investigate tattoo-associated skin infections due to Mycobacterium chelonae using Emergency Department (ED) syndromic surveillance.

\section{Introduction}

In 2012, an outbreak of Mycobacterium chelonae infections in tattoo recipients in Rochester, NY was found to be associated with premixed tattoo ink contaminated before distribution. ${ }^{1}$ In May 2012, a case of M. chelonae was reported in a New York City (NYC) resident who received a tattoo with ink alleged to have been diluted with tap water. When a second case of $M$. chelonae in a tattoo recipient was reported in March 2013, an investigation was initiated.

$M$. chelonae is not reportable in NYC other than in clusters reported by providers or laboratories. To determine if there were additional tattoo-associated M. chelonae infections, we searched for cases using NYC ED syndromic surveillance.

\section{Methods}

ED syndromic data is de-identified and received daily from 49 of the 52 acute care hospitals in NYC. ${ }^{2}$ Patient chief complaints are routinely scanned for key words and coded into syndromes (e.g. influenza-like illness, asthma). Chief complaint data containing the key word "tattoo" for the period January 1, 2008 - December 31, 2012 were selected for analysis. The data were analyzed to describe trends and identify ED visits suspicious for skin infection (chief complaints containing the words "cellulitis," "infected," "redness," etc.).

ED visits that met our criteria in the five months preceding the recent $M$. chelonae report were selected for interview (November 2012-March 2013). Names and contact information associated with the chief complaint data were obtained from hospital staff. A questionnaire including symptoms and duration, location of tattoo, name of tattoo parlor, and artist information was administered to evaluate possible M. chelonae cases requiring referral to a dermatologist for diagnosis. NYC laboratories were contacted to inquire about skin or soft tissue cultures from 2012 or 2013 in which M. chelonae was isolated.

\section{Results}

A total of 577 tattoo-related ED visits (TREDV) representing 43 (88\%) hospitals were identified during 2008-2012. A $26 \%$ increase in the proportion of TREDV per total ED visits among ages 18-64 was noted from 2008-2012. Chi square for trend was not significant $(\mathrm{p}=0.11)$. Three-hundred eighty $(66 \%)$ of these visits were identified with additional chief complaint wording suggestive of infection.

Thirty-one TREDV were identified in the five-month period preceding the second reported case of $M$. chelonae. ED visits were distributed among 19 NYC hospitals (range 1-4 visits/hospital). The median age of patients was 24 (range 16-48) and 65\% (20) were women. For $18(58 \%)$ patients, the chief complaint was coded as infection, $10 \%$ (3) for rash, $10 \%$ (3) for swelling, $7 \%$ (2) for pain and $16 \%$ (5) for other (allergic reaction, redness, warmth, not specified).

Interviews were conducted for $14(45 \%)$ of the TREDV. Reasons for unsuccessful interviews included no reply to three phone call attempts and wrong or disconnected phone numbers. ED patients interviewed did not differ significantly from those who could not be reached on age, sex, or borough of residence. Thirteen $(93 \%)$ interviewed patients had resolution of symptoms or a noninfectious diagnosis. One patient who had persistent symptoms was referred for additional medical care and was diagnosed with Staphylococcus aureus.

From January 2012 through March 2013, NYC laboratories reported 13 isolates of M. chelonae from skin or soft tissue specimens, none of which were from patients with recent tattoos.

\section{Conclusions}

Using ED syndromic surveillance data, we determined that one case of tattoo-related $M$. chelonae was not part of an unrecognized outbreak or cluster of cases. In response to this concern about $M$. chelonae infections in tattoos, the Health Department sent a letter to all licensed tattoo artists in New York City advising them not to dilute tattoo inks with tap water. Syndromic surveillance is an option for finding cases when the event under surveillance is described by a unique and specific word or phrase, such as tattoo. This method can be similarly used for situations where diseases are either not reportable or when cases may be otherwise difficult to capture.

\section{Keywords}

tattoo; Mycobacterium chelonae; syndromic surveillance

\section{References}

1. Giulieri S, Cavassini M, Jaton K. Mycobacterium chelonae Illnesses Associated with Tattoo Ink. N Engl J Med. 2012; 367(24): 2357-8.

2. Heffernan R, Mostashari F, Das D, Karpati A, Kulldorff M, Weiss D. Syndromic Surveillance in Public Health Practice, New York City. Emerg Infect Dis. 2004; 10(5):858-864.

\section{*Mollie Kotzen}

E-mail: mkotzen@health.nyc.gov 